\title{
Norois
}

Environnement, aménagement, société

\section{Diffusion d'une culture alimentaire régionale etrestauration : Kii Tanabe (Japon)}

Diffusion of a regional food culture and restaurants: Kii Tanabe (Japan)

\section{Sylvie Guichard-Anguis}

\section{(2) OpenEdition}

1 Journals

\section{Édition électronique}

URL : https://journals.openedition.org/norois/3588

DOI : $10.4000 /$ norois.3588

ISSN : 1760-8546

\section{Éditeur}

Presses universitaires de Rennes

\section{Édition imprimée}

Date de publication : 30 juin 2011

Pagination : 23-39

ISBN : 978-2-7535-1479-9

ISSN : 0029-182X

\section{Référence électronique}

Sylvie Guichard-Anguis, « Diffusion d'une culture alimentaire régionale etrestauration : Kii Tanabe (Japon) », Norois [En ligne], 219 | 2011, mis en ligne le 30 septembre 2013, consulté le 14 janvier 2022. URL : http://journals.openedition.org/norois/3588 ; DOI : https://doi.org/10.4000/norois.3588

Ce document a été généré automatiquement le 14 janvier 2022.

(ㄷ) Tous droits réservés 


\title{
Diffusion d'une culture alimentaire régionale etrestauration : Kii Tanabe (Japon)
}

\author{
Diffusion of a regional food culture and restaurants: Kii Tanabe (Japan)
}

\author{
Sylvie Guichard-Anguis
}

1 Cet article ne concerne pas un (grand) restaurant tel que l'on en trouve dans les provinces françaises car tout simplement ce type d'établissement n'existe pas sous cette forme au Japon. Les modes de consommation alimentaire japonais présentent en effet des différences sensibles dont l'analyse va permettre de mettre en valeur la spécificité culturelle de ce type d'établissement à la française. L'extraordinaire richesse gustative de l'archipel japonais s'appuie sur un ensemble d'établissements extrêmement variés qui forme un tout à l'échelon local, voire régional et dont aucun n'est perçu comme se distinguant tout particulièrement. Concernant la péninsule de Kii, la plus grande au Japon où se trouve Kii Tanabe la ville sur laquelle porte cette étude, aucun des responsables de la promotion touristique des différents départements concernés n'a pu citer un seul nom d'établissement se détachant du lot, car tout simplement cette question ne peut se poser au Japon, tant les réponses sont multiples.

2 Afin de forcer le trait de cette démonstration, le choix s'est porté sur cette petite ville (dans le contexte japonais) loin des grands foyers gastronomiques comme Tokyo ou Kyoto, de manière à démontrer à quel point cette culture pénètre le moindre recoin de l'archipel. En d'autres termes, de grands établissements se détachant de ces ensembles locaux déjà si richement pourvus ne peuvent se trouver jusqu'à présent, compte tenu des modes de consommation gourmands en usage.

Dans un premier temps, nous allons constater qu'au Japon les déplacements accompagnés d'une consommation gourmande s'inscrivent dans des habitudes qui remontent à plusieurs siècles et que ces dernières continuent à conditionner le présent. Une rapide présentation permettra ensuite de mieux connaître Kii Tanabe, petite ville qui a priori semble bien loin de constituer un foyer gastronomique mais qui se projette progressivement sur la scène touristique internationale. La richesse de l'offre locale 
contemporaine nous permettra de comprendre en quels termes elle se présente un peu partout au Japon et freine l'émergence de (grands) restaurants à la française.

\section{Déplacements et consommation gourmande au Japon, une relation déjà ancienne de plusieurs siècles}

4 Le phénomène d'attraction exercé en France par des établissements de renommée internationale, situés dans des zones rurales à l'écart des grandes villes n'a pas de véritable équivalent au Japon. Seuls quelques restaurants peuvent prétendre s'apparenter à ce phénomène mais leur réputation reste encore loin de s'exercer sur le plan national, voire international et surtout ils ne se trouvent jamais totalement à l'écart d'un centre touristique. Apparus vers la fin des années 1990, ces établissements se spécialisent dans l'offre d'une cuisine française, voire italienne de grande qualité, proposées dans un environnement qui tend à suggérer celui qui est susceptible de se trouver dans ce genre de restaurants en France. Citons l'«Ermitage de Tamura» (Erumitāje dō Tamura) ouvert en 2000 dans l'ancienne résidence secondaire d'un écrivain, à proximité de la station estivale de montagne à Karuizawa. Tenue par un chef cuisinier venu de Tokyo, elle propose une cuisine française légère afin de contredire la réputation de cette dernière, qui fait allusion à la sempiternelle présence de sauces très riches. Le phénomène reste cependant très récent et marginal et surtout n'a pas du tout l'impact que peuvent avoir les grands restaurants français sur le renouvellement de la perception de certaines régions rurales. L'offre d'une cuisine européenne dans un milieu rural, pratique inhabituelle pour un restaurant d'un certain niveau au Japon, ajoute à la sensation d'étrangeté, d'invitation au voyage et de ressourcement au sein d'une nature que le visiteur dégustera en partie dans son assiette. Il est à noter qu'il ne s'agit pas de cuisine japonaise, qui elle prend son sens dans un tout autre environnement comme nous allons le constater plus loin.

5 L'une des raisons principales de cette absence réside dans une concurrence très vive du fait d'un remarquable foisonnement d'établissements de qualité, qui plonge ses racines dans les périodes précédentes à partir de celle d'Edo (1603-1868). En effet depuis des siècles les déplacements s'associent à des expériences gourmandes dans des endroits parfois fort isolés. La littérature de voyage particulièrement riche au Japon et forte d'œuvres célèbres qui s'étalent sur un peu plus d'un millénaire s'en fait largement l'écho comme dans le très célèbre roman "À pied sur le Tōkaidō» (Tōkaidōchu hizakurige) publié en 1806 (Jippenshā, 1992). Analyser rapidement ces relations entretenues depuis des siècles entre le voyage et les consommations gourmandes paraît indispensable tant elles continuent à conditionner les déplacements gourmands dans le Japon de ce début du second millénaire.

6 Jusqu'à la fin du XIX $x^{e}$ siècle les habitants de l'archipel japonais circulent à pied et, fait tout à fait remarquable, dans des proportions sans équivalent dans l'Europe d'alors. On estime qu'une personne sur vingt accomplit le pèlerinage aux grands sanctuaires d'Ise (actuel département de Mie) vers le milieu de l'époque d'Edo (Kanzaki, 2002). Cette mobilité se base sur la présence de deux capitales (impériale et féodale) séparées de plus de $350 \mathrm{~km}$ entre lesquelles les relations sous toutes leurs formes doivent être entretenues constamment. La division de l'archipel en près de 300 fiefs, sans oublier les régions administrées directement par le shogounat, résulte en mouvements supplémentaires entre les différents centres féodaux et ces capitales. L'obligation pour 
les seigneurs (daimyo) et leurs principaux vassaux de vivre en alternance (Vaporis, 2008) entre " la ville en bas du château » (jōka-machi) centre du fief et Edo contraint à la circulation de processions de plusieurs centaines d'hommes voir milliers régulièrement le long des artères (kaidō). Outre toutes les activités associées aux échanges de toutes sortes, la population japonaise circule à des fins récréatives sous des prétextes religieux (principalement les pèlerinages, comme celui d'Ise etc.) ou thérapeutiques (séjour dans des stations thermales onsen) (Guichard-Anguis, 2008a). Un réseau routier qui se met en place dans la première moitié du XVII ${ }^{e}$ siècle permet de joindre les principaux points de l'archipel. Les cinq principales routes (go-kaidō) à partir d'Edo en sont les plus célèbres représentantes et les plus illustrées par l'estampe. Des villes étapes (shukuba-machi), pourvues d'auberges de différentes catégories (Guichard-Anguis, 2008b) jalonnent ces artères. Leur fréquence le long des principaux axes permet de comprendre en partie le foisonnement contemporain des établissements de restauration. En effet, cas inhabituel dans un pays développé, certaines portions de ces routes subsistent toujours, du fait de leur simple abandon à la suite de la construction de voies routières à l'écart, ou bien de leur transformation en axe routier secondaire.

Outre l'hébergement et la fourniture d'articles divers destinés à la poursuite du voyage, ces villes étapes se dotent d'établissements où l'on peut se restaurer rapidement. Si le séjour dans les auberges comporte pour les plus aisées d'entre elles le repas du soir et du matin avant le départ au lever du soleil (nous y reviendrons), des chaya permettent de reprendre vite des forces dans la journée. Les chaya (lit. établissements de thé) se situent tout au long de ces voies, que ce soit au cœur d'une ville étape même ou isolés au sommet d'un col et le Japon n'en manquent pas! L'offre d'un gobelet rempli de thé vert (Kozu, 2009) accompagné d'une douceur de la taille d'une grosse bouchée et posée sur une petite assiette permet au voyageur épuisé par sa marche de reprendre quelques forces avant de poursuivre son chemin (Guichard-Anguis, à paraître). Elles soulignent le lien indissociable entre la consommation du thé vert et celle des produits sucrés d'origine japonaise, qui se poursuit toujours dans le Japon du xxI siècle. Cette prise alimentaire s'effectue en dehors des repas principaux. Cette habitude reste la règle de nos jours et explique la présence d'un grand nombre d'établissements uniquement spécialisés dans cette offre. Certains de ces chaya offrent du saké (Kanzaki, 2002) dont la teneur en sucre permet également de redonner de l'énergie au marcheur. Un certain nombre de ces chaya remontant à plusieurs siècles subsiste et constitue un but touristique contemporain. À Uji, Tsūen chaya remonterait au moins au XvI ${ }^{\mathrm{e}}$ siècle, ce qui en ferait la plus ancienne du pays. En 2010 elle propose différents assortiments de thé et de douceurs, quelques plats de pâtes et mets glacés ${ }^{1}$.

8 Bon nombre de ces douceurs appartiennent aux spécialités locales meibutsu, dont certaines remontent à plusieurs siècles. Ces dernières ne cessent de se multiplier de nos jours exerçant le même attrait en ce début de $\mathrm{xxI}^{\mathrm{e}}$ siècle, un lieu attractif ne pouvant exister dans l'imaginaire sans cette icône. Offertes par toutes sortes de commerce alimentaire, elles constituent un facteur attractif non négligeable qui concourt à la dispersion des prises alimentaires. La douceur Aka-fuku, qui remonterait à 1707, reste malgré un récent scandale associé à sa fraîcheur dont tout le pays s'est fait l'écho, indissociable du pèlerinage à Ise susmentionné ${ }^{2}$. Elle peut se consommer sur place lors de la visite aux Grands sanctuaires Naikū, ou bien être rapportée dans des emballages adéquats. Ces spécialités peuvent trouver leurs origines dans des ingrédients terrestres ou marins, des savoir-faire, une légende, une histoire, des rites etc. tous profondément 
ancrés dans les lieux. Nombre d'entre elles qui sont apparues au cours de l'époque d'Edo, en particulier le long de ces axes restent toujours aussi connues. Leur densité devient telle que l'on peut organiser une exposition comme celle qui s'est tenue dans la galerie de la grande maison de douceurs Toraya au siège à Tokyo en 2006 (Toraya Bunko, 2006). Elle reproduisait quelques-unes des douceurs locales décrites dans des journaux de voyage de l'époque d'Edo.

9 Ces spécialités peuvent faire l'objet d'un achat afin de les rapporter sous forme de cadeaux souvenirs (omiyage) aux proches qu'ils soient de la famille, du lieu de travail ou de tout groupe auquel appartient le visiteur (Kanzaki, 1997), ou bien être consommées sur place, et même souvent faire l'objet des deux démarches. Se rendre dans un lieu précis, c'est toujours au Japon consommer quelque chose de ce lieu, qu'il soit sucré ou bien sous forme de repas rapide. L'offre de plats de pâtes de sarrasin lié à un environnement peu favorable à la riziculture et le Japon n'en manque pas, diffère invariablement par quelque procédé d'une région à l'autre de l'archipel. Ces spécialités selon leur nature peuvent soit être consommées seules sous forme d'encas ou de déjeuner ou bien se trouver inclus dans le menu du soir proposé par les auberges japonaises de type ryokan qui proposent invariablement un hébergement accompagné du dîner et du petit-déjeuner.

10 La consommation de ces spécialités locales, souvent anciennes, participe au phénomène d'identification aux lieux et à un passé qui se révèle le plus souvent une reconstruction et parfois une invention d'un temps jadis idéalisé face à un Japon qui s'uniformise à grande allure. Se rendre dans ces établissements au début du XXI ${ }^{e}$ siècle autorise à se plonger brutalement et temporairement dans un ailleurs situé dans le temps pour les visiteurs japonais contemporains. Face à l'hyper modernité en construction permanente dans le Japon urbain, les visiteurs japonais éprouvent le besoin de revenir dans un univers avec lequel ils ont perdu toute familiarité mais qui, finalement, remonte à un passé pas si lointain. Les prises alimentaires, en particulier à travers la consommation de meibutsu jouent un rôle considérable dans ce mécanisme.

11 Sans doute est-ce aussi en partie la raison pour laquelle, fait assez exceptionnel dans le monde, deux types d'hébergements continue à exister côte à côte au Japon. Parallèlement au courant d'origine occidentale associé aux hôtels et apparu à la fin du $\mathrm{XIX}^{\mathrm{e}}$ siècle, coexiste au Japon un autre type d'hébergement rattaché aux auberges japonaises (Nihon no yado), qui plonge ses racines dans un passé plus lointain, au moins dans celui de l'époque d'Edo (Guichard-Anguis, 2003a). L'expression «auberges japonaises » fait allusion à tous les types d'hébergement apparus dans l'archipel japonais et qui ne tirent pas leur origine de la tradition hôtelière. Les auberges de type ryokan en constituent la grande majorité.

12 Si dans la journée le visiteur a tendance à consommer sous forme de différentes prises alimentaires en fonction de ce qu'offrent les lieux, le soir le dîner s'effectue dans le cadre d'une auberge japonaise, ce qui contribue à l'extraordinaire émiettement des établissements. De nos jours il s'avère de plus en plus difficile d'établir une limite claire entre l'univers hôtelier et celui des auberges qui ne cessent de s'influencer mutuellement. Cependant l'une des différences fondamentales continue à résider dans l'offre d'une pension semi complète dans le second cas. En effet l'un des facteurs essentiels d'attractivité des auberges japonaises consiste dans la cuisine proposée, conjuguée au bain et à la qualité de l'hospitalité (Guichard-Anguis, 2003b). Malgré la crise sévère qui frappe ce secteur de l'activité économique, le Japon compte encore 
58000 auberges (Japan National Tourist Organization) ce qui permet de comprendre la dispersion considérable de l'offre gastronomique le soir lors de déplacements de type touristique. Bref, dans la perception générale que l'on a de l'hébergement touristique au Japon, on associe généralement un bon repas à une nuitée. Une enquête effectuée auprès de ses lecteurs par le grand quotidien Asahi à travers un de ses sites internet reste très révélatrice à ce propos (Asahi, 2009). Prendre son repas dans sa chambre représente la seconde raison après le bain pour laquelle l'auberge est préférée à l'hôtel, et le repas constitue le second critère de choix après la présence de bains thermaux. Quant à l'hôtel, ne pas être prisonnier des horaires de repas et rester libre dans ce domaine constituent les deux premières raisons de leur choix, tandis que le prix joue comme premier critère. La dictature des horaires de repas exercée par les auberges japonaises (le dîner se prend entre $18 \mathrm{~h} 00$ et $19 \mathrm{~h} 00$ et le petit-déjeuner entre 7 h00 et 8h00) tend lentement à s'alléger. Le voyageur japonais contemporain beaucoup plus individualiste supporte de moins en moins ces contraintes parfaitement adaptées aux groupes touristiques qui ont fait les grandes heures de ces auberges dans les années 1960 jusqu'au milieu des années 1970.

\section{Le boom du gourmet et la redécouverte de la cuisine locale à des prix raisonnables}

13 Un autre facteur de la dispersion de la consommation gourmande tient au menu luimême, inexistant ou ne comportant pratiquement pas de produits sucrés. En effet, d'une part une très grande partie des établissements de restauration au Japon est très spécialisée, sur un seul produit (la cuisson d'anguille par exemple) ou sur un mode de préparation (la cuisson sur une plaque chauffante). D'autre part, le menu tel qu'il a été adopté dans les restaurants urbains de luxe (ryōtei) ou les ryokan tire son origine de celui servi lors de réunions dans le cadre de la pratique de la cérémonie de thé (chanoyu). Ce menu kaiseki dont la structure s'est élaborée au cours des siècles, voit se succéder dans un ordre presque invariable un certain nombre de mets qui à chaque fois se distingue par leur préparation (cru et coupé, cuit au court-bouillon, etc.), à toutes sortes de préparations liquides. Tout ce qui se rapporte au sucre se consomme généralement en dehors des repas avec le thé, même si l'usage contemporain privilégie quelques fruits frais et glaces à la fin du repas. Ceci explique que l'on peut tout naturellement diviser sa consommation gourmande dans différents établissements au cours de la journée.

Enfin il faut terminer cette très rapide présentation du contexte par l'évocation du «Boom du Gourmet » (Gurume no būmū) qui caractérise les deux dernières décennies du $\mathrm{Xx}^{\mathrm{e}}$ siècle pour se poursuivre toujours de nos jours (Ashkenazy et Jacob, 2000). La transcription du mot français "gourmet» en japonais, traduit en général par «le mangeur qui marche » (tabe aruki) est très significative de l'évolution de ce terme dans le contexte japonais. Du petit matin jusqu'au milieu de la nuit, il n'y a pas d'heures sans programme de télévision montrant un voyageur à l'intérieur de l'archipel découvrant les spécialités locales. Il arrive toujours à pied au lieu de destination de la dégustation, que ce soit celui de la prise de pêche, le champ où est cultivé un légume ou fruit spécifique ou bien le petit établissement où est cuisinée la spécialité qui fait l'objet du programme. Ces découvertes gourmandes régulièrement ponctuées de dégustations, font l'objet d'invariables gros plans et s'achèvent par la sempiternelle exclamation 
«oishi » (c'est délicieux). Non seulement les chaînes de télévision mais aussi l'ensemble des media couvrent ce domaine de manière intensive, jusqu'aux bandes dessinées dont la plus célèbre reste sans conteste le Gourmet solitaire (Taniguchi et Kusumi, 2005). En d'autres termes le Japon contemporain se caractérise par l'évocation omniprésente du plaisir de bien manger et offre une diversité dans ce domaine assez exceptionnelle car cette gourmandise s'étend désormais à toutes les cuisines du monde, aspect que nous ne pouvons développer dans ces lignes.

Nement intitule «Okayama B kyu fuesuta in Tsuyama" " qui se traduit par "Fête de niveau B à Tsuyama dans Okayama " s'est déroulé les 20 et 21 mars 2010 à Tsuyama (département de Okayama). Le choix du concept de niveau $\mathrm{B}$ fait allusion à la définition de ces mets qui doivent comporter invariablement les caractéristiques suivantes. Ils doivent être économiques, délicieux, faciles à exécuter. Fortement associés au renouveau local (machi okoshi) ils doivent tirer partie des savoir-faire et des produits locaux. Ils se définissent par rapport à une cuisine élitiste, peu abordable par la grande majorité de la population et qui sans doute se positionnerait au niveau A. Ce mouvement fondé par un journaliste gastronomique ne cesse de prendre de l'ampleur ces dernières années et repose essentiellement sur l'action de bénévoles. La grande majorité de ces mets date de la seconde moitié $\mathrm{du} \mathrm{xx}^{\mathrm{e}}$ siècle, ce qui souligne l'extraordinaire inventivité et renouvellement de la cuisine japonaise. Les mets les plus prisés se classent parmi ceux de pâtes de type ramen (d'origine chinoise), les sushi proposés en boucle (kaiten zushi), les petits pâtés de viande frits à la chinoise (gyoza), les grillades d'abats (horumon yaki) etc.

Durant cette manifestation qui a attiré plus de 150000 visiteurs en deux jours, cinquante-quatre plats différents provenant de tout l'archipel étaient proposés. Parmi eux se trouvait le curry de Bizen (Bizen karē) qui comporte une nouvelle spécialité (shin meibutsu) : du bœuf mijoté dans une sauce épicée à base de confiture de figues, servi sur un bol de riz blanc (donburi). N'oublions pas que le karē qui a perdu pratiquement toute ressemblance avec les mets d'origine, reste l'un des plats familiaux favoris au Japon.

\section{Kii Tanabe une petite ville dans une région en voie de désertification}

17 Avant d'aborder la culture alimentaire de Kii Tanabe au sein de ce très riche foisonnement, une rapide présentation de cette petite ville s'impose. Kii Tanabe est située dans la péninsule de Kii la plus grande du Japon, à une centaine de kilomètres au sud de Osaka (fig. 1).

Malgré une situation qui peut sembler quelque peu périphérique au premier coup d'oeil, son éloignement des grands centres névralgiques de l'archipel est compensé par la présence de l'autoroute qui s'arrête dans la municipalité voisine juste au nord mais devrait être prolongée (...dans un avenir proche sans cesse repoussé). La voie ferrée qui longe sur la côte cette presqu'île dessert beaucoup mieux la partie orientale où se trouve Kii Tanabe qu'occidentale vers Nagoya. L'aéroport de Nanki Shirahama juste au sud dans la commune voisine dessert deux fois par jour Tokyo (et trois fois pendant la période estivale) mais son avenir s'est assombri avec la situation très difficile de la compagnie aérienne nationale japonaise JAL. Cependant au nord dans la partie méridionale de la préfecture urbaine d'Osaka se trouve l'aéroport international du 
Kansai, relié par des bus express qui empruntent l'autoroute. En résumé Kii Tanabe se trouve désormais à deux heures de route du Kansai la région d'Osaka, Kyoto, Nara et Kobe et une heure par avion de la capitale Tokyo. Cette proximité nouvelle influence les choix de développement de la ville contemporaine et en particulier ceux associés à l'attraction touristique.

La présence des liaisons de première importance uniquement sur la côte suggère une difficulté de circulation à l'intérieur de la presqu'île. Il s'agit d'une région de montagnes qui comprend 17 cols de plus de mille mètres et dont $81 \%$ des pentes possèdent une déclivité supérieure à $30 \%$. Ce relief dicte des modes d'occupation et par là même des modes de vie en rapport direct avec la culture alimentaire. L'absence de véritable plaine et la grande difficulté d'accès toujours manifeste au $\mathrm{xxI}^{\mathrm{e}}$ siècle par rapport à bon nombre d'autres régions de l'archipel n'a pas permis le développement de grandes villes et Kii Tanabe se range parmi les principales villes qui sont apparues le long de cette côte.

La seconde constatation à la lecture de cette carte provient de la taille de la commune de Kii Tanabe au sein du département de Wakayama. Elle occupe un quart de celle du département : $1026 \mathrm{~km}^{2}$ pour $4725 \mathrm{~km}^{2}$ (sources municipales). Elle s'étend sur $45 \mathrm{~km}$ du nord au sud et $46 \mathrm{~km}$ d'est en ouest. Le cœur urbain de l'ancienne municipalité de Kii Tanabe se trouve dans la partie occidentale sur la côte tandis que le reste fait partie de la région montagneuse de Kii. Depuis 2005 date de la dernière fusion, la commune actuelle comprend outre celle originelle de Kii Tanabe les villages de Ryūjin et Ōto, les bourgs de Nahahechi et Hongū. Cette dernière fusion joue un rôle fondamental sur les nouvelles orientations prises par la municipalité, puisque désormais avec le bourg de Hongū, des éléments figurant sur la liste du patrimoine mondial de l'Unesco se trouvent incorporés au territoire communal. Sur ce dernier seuls 3,4\% des terres sont consacrées à l'agriculture et 1,2\% urbanisées contre $89,3 \%$ occupés par les pentes forestières des montagnes (les 6,1 \% relevant de divers : routes, voies d'eau, etc.). 
Figure 1 : Carte de localisation de Kii Tanabe, Japon/Location map (Kii Tanabe, Japan)

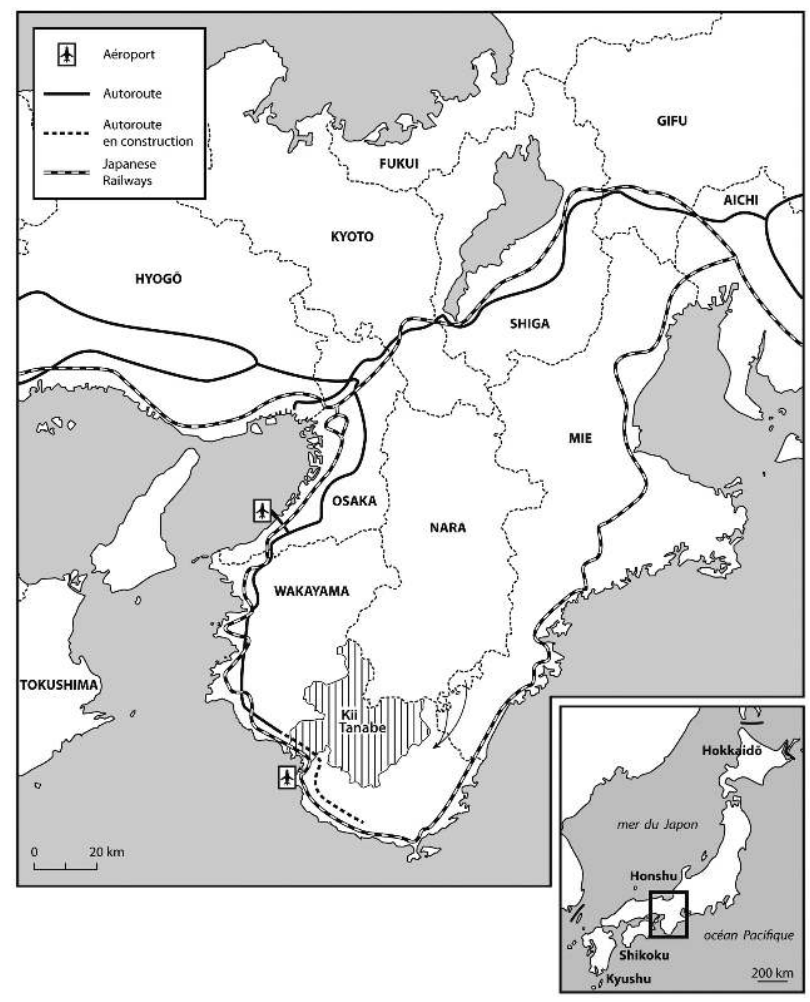

21 Une telle répartition ne peut influencer que fortement les ressources alimentaires disponibles et un seul coup d'œil permet de comprendre que la commune de Kii Tanabe ne s'inscrit pas du tout dans le Japon rizicole. De façon générale ce dernier est toujours resté minoritaire du point de vue des superficies occupées. Au contraire cette commune offre un exemple des stratégies mis en place pour survivre dans le Japon des régions montagneuses. Le climat traduit cette très forte disparité puisque sur le littoral caractérisé par un climat chaud et pluvieux de type océanique associé à la présence de l'Océan Pacifique, succède un climat plus continental et plus froid. Concernant la pluviosité ils tombent $1638 \mathrm{~mm}$ de pluie à Shirahama contre $2749 \mathrm{~mm}$ à Ryūjin. La région montagneuse de Kii compte quelques-unes des zones les plus arrosées du Japon avec plus de $3000 \mathrm{~mm}$.

En 2006, la population de l'ensemble de la commune de Kii Tanabe s'élevait à 82499 habitants, et l'ancienne ville de Tanabe à 68117 habitants. Cette commune se trouve au sixième rang du département de Wakayama, l'un des plus faiblement peuplé de l'archipel. Si la population de l'ancienne Kii Tanabe représente 82,2\% de celle de la commune et se maintient tant bien que mal, le reste du territoire se caractérise par la désertification et un vieillissement très rapide. L'exode rural qui a commencé dans les années 1950, a pratiquement vidé ces régions montagneuses, dans lesquelles les hameaux et les fermes abandonnés ne manquent pas. La répartition de la population active souligne encore mieux ce phénomène. Le secteur primaire rassemble $14,4 \%$ contre $5 \%$ en moyenne nationale, le secteur secondaire $23 \%$ contre $29,5 \%$, et le secteur tertiaire $62,1 \%$ contre $64,3 \%$. Outre le secteur agricole, l'importance de la sylviculture sur le territoire municipal contemporain explique un tel chiffre, qui donne des traits bien particulier à cette commune dont $90 \%$ du territoire reste occupé par la forêt. 

petite ville située dans une région quelque peu déshéritée. En 2004 « les lieux sacrés et les chemins de pèlerinage de la région montagneuse de Kii » ont été incorporés sur la liste du patrimoine mondial de l'Unesco. Des chemins relient à travers les montagnes de la région de Kii des lieux sacrés apparus avant la fin du premier millénaire, indépendamment les uns des autres. Vers la fin $d u x^{e}$ siècle avec la formation $d u$ syncrétisme shintō bouddhiste les trois grands ensembles sacrés les plus au sud en sont venus à former un tout sous le nom de trinité de Kumano (Kumano sansho gongen). À la même époque les divinités à l'origine shintoïste de ces sanctuaires ont été assimilées à des manifestations locales de bouddha et confèrent à cette région le statut de sacré associé à la notion de paradis bouddhique. Ce paradis présent dans les montagnes de Kumano, s'étend bien au-delà dans la mer du sud qui borde le rivage (Moerman, 2006).

nos jours Kii Tanabe constitue la porte d'entrée de la portion de chemin de pèlerinage la plus populaire $\mathrm{du}$ "chemin du milieu» Nakahechi, qui venant de l'ancienne capitale impériale devenue Kyoto, passe par l'actuelle Osaka, suit la côte et bifurque pour pénétrer dans les montagnes à proximité de Kii Tanabe (Koyama, 2004 ; Koyama, 2000). Il permet de se rendre à Kumano Hongū Taisha le premier des trois grands sanctuaires de Kumano que rencontre le visiteur lors de son pèlerinage. De là, il peut se rendre jusqu'à Kumano Hayatama Taisha sur la côte et ensuite à Kumano Nachi Taisha avant de remonter vers Kumano Hongū Taisha et de revenir dans le Kansai par Kii Tanabe. Les visiteurs actuels tendent à préférer un simple aller vers Kumano Hongū Taisha, à partir de Kii Tanabe où ils descendent du train pour prendre un bus qui les mènera à l'intérieur des montagnes, et de là ils commencent à marcher.

Ces chemins empruntés depuis plus d'un millénaire par toutes les couches de la société et richement documentés à travers une littérature de voyage qui continue toujours de nos jours à s'enrichir, deviennent aujourd'hui des itinéraires de marcheurs. Depuis la nomination sur la liste de l'Unesco, toutes les collectivités locales concernées tentent de bâtir leur avenir sur la promotion touristique de ce pèlerinage comme celui de la visite aux grands sanctuaires. Kii Tanabe possède un bureau de tourisme extrêmement actif dans ce domaine qui emploie un étranger d'origine canadienne, de façon à promouvoir ces chemins sur le plan international.

\section{La région montagneuse de Kii : pénurie et richesse culinaire}

Dans un tel contexte la pénurie et l'autosubsistance ont longtemps dominé la culture alimentaire de Kii Tanabe et de la région environnante. L'un des plats emblématiques de cette région montagneuse reste toujours le gruau de riz au thé (cha gayu). Pendant des siècles en particulier à l'époque d'Edo des taxes très élevées privant les paysans de l'essentiel de leur récolte en riz se sont surimposées à la rareté des surfaces planes. L'une des trois grandes familles Tokugawa dont sont issus les shogun de l'époque d'Edo se trouve dans le fief de Kishū qui correspond en partie à l'actuel département de Wakayama. Afin de soutenir les dépenses de ce fief de première importance des taxes très lourdes sont prélevées sur les paysans. Elles se calculent en riz suivant un système qui évalue la capacité productive des terres en boisseaux de riz (kokudaka), cette céréale constituant l'unité de revenu de la classe guerrière. Les rizières abandonnées en étages au cœur des montagnes ne manquent pas en ce début de millénaire et traduisent la 
sévérité de ces conditions extrêmes de vie. De façon générale, il faut rappeler que l'on s'est mis à consommer du riz blanc trois fois par jour au Japon qu'après la Seconde Guerre mondiale : jusque-là cette céréale représentait une denrée trop onéreuse pour être servie aussi régulièrement. Le thé de Kishū représente une toute petite production par rapport aux grandes régions spécialisées dans cette culture. Cependant jusqu'à une époque très récente des théiers plantés à proximité des habitations fournissent du thé destiné à la consommation domestique. Ces feuilles de thé peuvent se préparer également en beignets (tempura), un mets plus contemporain compte tenu des ingrédients utilisés (œuf et farine). Pour faire du gruau de thé, il suffit d'ajouter à l'eau chaude des feuilles de thé vert Bancha (thé de seconde cueillette et des suivantes) légèrement fumé, enfermées dans un sac. On verse ensuite le riz dans cette infusion. Il cuit en éclatant ce qui donne l'illusion d'un plus grand volume par rapport au riz cuit dans une mesure d'eau adéquate. Des patates douces peuvent être ajoutées à ce gruau afin de renforcer son apport énergétique. Ce mets se consomme brûlant avec quelques légumes en saumure (tsukemono). Désormais il incarne une spécialité touristique, une icône de la culture alimentaire régionale contemporaine.

Ce renversement de tendance d'un met emblématique de la frugalité devenu une spécialité gastronomique ne se cantonne pas au Japon et se trouve finalement assez répandu dans les régions développées. Cependant le contraste entre l'abondance contemporaine et l'état d'auto subsistance pratiquement général d'avant-guerre se trouve particulièrement fort dans cette région. Un de nos interlocuteurs rencontré à Shingū né dans les années 1930 dans un hameau isolé au-delà de Koguchi nous racontait qu'il a attendu l'âge de 6 ou 7 ans, soit le début des années 1940 pour voir pour la première fois de l'argent. Les seules viandes que sa famille consommait provenaient de la forêt avec celles de cerfs et sangliers.

Une étude récente offre un aperçu très suggestif de cette distance. Elle provient d'une série de recherches collectives menées depuis $1996^{4}$ par des sociologues principalement de l'université de Kyoto. Elles analysent d'année en année les différents hameaux ou villages de la commune de Kumano qui se trouvent de l'autre côté de la péninsule de Kii. Cette dernière, située dans le département de Mie compte 20105 habitants en 2009 sur un territoire de $373,63 \mathrm{~km}^{2}$ qui s'étend de l'Océan Pacifique à la région montagneuse de Kii. Un des chemins de pèlerinage vers les grands sanctuaires de Kumano, celui de Ise Iseji la traverse. Elle possède les mêmes caractéristiques que la commune de Kii Tanabe avec un territoire occupé par la forêt à $88 \%$, en voie de désertification (elle comptait 22640 habitants en 2000) et doté d'une population qui vieillit très rapidement. Kodokoro hameau de l'ancien bourg Ikusei dans la partie montagneuse au sud-ouest de l'actuel territoire communal de la ville de Kumano a été incorporé récemment à cette dernière. Une étudiante en sociologie a rédigé cette étude comme toutes celles qui composent le volume, dirigé par des professeurs des universités de Hōsei, Kansai et Kyoto. Ce travail couvre une période qui s'étend des années d'aprèsguerre jusqu'au milieu des années 1970.

Après avoir démontré le rôle central dans les rites shinto du riz, des mochi ${ }^{5}$, du sake, fait assez général dans les hameaux de l'archipel et du scombrésoce (sanma) un poisson qui se trouve en abondance localement, l'auteur d'une vingtaine d'années aborde la culture alimentaire locale de façon générale. Elle qualifie les menus de « repas traditionnels de jadis ", en insistant sur le fait qu'ils se rangent dans la catégorie washoku (cuisine japonaise «traditionnelle » par opposition à la cuisine japonaise d'origine occidentale) 
et sont intégralement faits maison. Elle note que ses interlocuteurs déplorent la disparition de toutes sortes de produits de cueillette ou de pêche fournis par la nature à la suite des bouleversements subis par l'environnement local. La construction de barrages hydro-électriques sur les cours d'eau avoisinant a entraîné la disparition des anguilles, des truites (ayu), des crabes de rivière etc. La sylviculture a fait disparaître tous les champignons et les plantes sauvages comestibles (dont on est particulièrement friand au Japon au début du printemps). Cependant grâce au progrès de l'agriculture la consommation du riz blanc est devenue quotidienne dans la première moitié des années 1950. Jusque-là c'était le gruau de riz au thé qui constituait l'aliment de base. Les théiers poussaient dans les champs incultes, sur les bords de rizières etc. Ce gruau comportait très peu de riz et surtout plusieurs sortes de patates douces, du potimarron et juste un peu de sel pour le goût. Le riz cuit de façon habituelle comprenait toujours une grande proportion de froment. La jeune auteur souligne ce renversement de tendance du riz au froment (mugi gohan) emblématique de la cuisine saine dans le Japon contemporain et autrefois ordinaire d'une bonne partie des foyers ruraux.

La viande provenait non seulement d'élevages de lapins, de volailles et des bœufs de labour mais surtout du produit de la chasse. Les faisans, les cochons sauvages et les cerfs ne manquent pas dans les forêts naturelles de Kumano. La viande de boucherie comme les poissons de mer commencent à entrer dans l'ordinaire des habitants de ce hameau vers la fin des années 1950. À cette même époque ces familles adoptent les mets caractéristiques de la culture urbaine populaire comme les feuilles de choux farcies (rōru kiabetsu) et le riz au curry (karē raisu). Composés aujourd'hui d'un à deux membres du fait du très rapide vieillissement de la population et de l'exode rural, ces foyers continuent à s'alimenter à base de produits locaux. Le jeune auteur fait remarquer le caractère idéal d'un tel régime dans le Japon contemporain, à base de légumes et céréales produits sur place, qui en outre restent à l'écart de tous les scandales de l'industrie agroalimentaire.

Ces recettes destinées à mettre en valeur des produits locaux font aujourd'hui figure de spécialités, que le visiteur vient rechercher pour leur rareté. L'ouvrage de bande dessinée (manga) consacré au département de Wakayama (Kariya et Hanasaki, 2009) se plait à énumérer tous ces plats qui tirent partie d'une espèce végétale ou d'un produit de la mer particulier. Ainsi les noix de kaya, un conifère (Torreya taxifolia) dont la consommation est rendue possible qu'après un traitement particulièrement long pour enlever l'amertume, faisaient partie de ces fruits de la terre que l'état de pénurie ne dédaignait pas. Aujourd'hui les visiteurs les apprécient comme une rareté en provenance d'une époque révolue qui se rattache à un Japon de jadis imaginaire. Dans cette péninsule, des feuilles vertes d'origine diverse permettent d'entourer le riz et de le conserver plusieurs heures tout en l'imprégnant de leur parfum. Citons le plus connu Mehari zushi devenu la spécialité locale par excellence qui illustre systématiquement toutes les brochures touristiques et qui inévitablement compose le bentō (pique-nique) du marcheur contemporain. Elle consiste à entourer le riz aggloméré en grosse bouchée, agrémenté de légumes voire de grains de sésame, d'une feuille de moutarde conservée dans le sel. Mais aussi des feuilles de framboisiers sauvages dans la région de Kushimoto au bord de l'Océan pacifique, ou de bananiers japonais (Bashō) près d'Arida permettent de façonner des sushi pressés qui se conservent quelques heures. Les nare zushi (sushi fermentés) à base de maquereaux montrent aussi une remarquable diversité. Le manga souligne cette grande richesse de préparations comme le génie culinaire local qui a su transformer en mets gastronomiques des produits ordinaires. 
Tous ces mets se retrouvent à la carte des établissements locaux contemporains, qui peuvent se spécialiser dans l'offre d'une de ses préparations. À noter que ce phénomène se rattache à une tendance assez générale face à l'uniformisation de l'alimentation contemporaine.

Ce manga auquel ont collaboré les bureaux de promotion touristique de cette région, tient aussi à rendre hommage à l'esprit d'initiative des habitants. En effet la fabrication de la sauce de soja à la japonaise trouverait ses racines à Yuasa, petite ville au nord du département où se trouvent plusieurs maisons fort anciennes. Le manga présente la maison Kadochō qui utilise des cuves en cryptomères (ou cryptomeria, arbre de la famille des conifères) de Yoshino qui remontent à plus de 170 ans. Il fait l'éloge des pêcheurs de Yuasa qui à l'époque d'Edo n'hésitaient pas à aller pêcher la sardine jusqu'au large de la péninsule de Bōsō qui ferme la baie de l'actuelle Tokyo vers l'est. Les sardines constituaient un engrais très prisé pour la culture du coton à Kawachi dans le sud de l'actuelle Osaka.

Curieusement toutes allusions à la consommation locale de chair de dauphin sont omises dans ces pages et celles en rapport avec la baleine restent discrètes. Or le département de Wakayama compte plusieurs anciens ports baleiniers comme Taiji (où a été filmé le documentaire dénonçant la pêche ou le massacre des dauphins suivant l'angle où se place le locuteur) ou Kii Katsura. Faut-il y voir l'influence de la polémique menée par des étrangers ? Le hasard en avril 2010 nous a fait apercevoir dans le panier d'un client d'une petite supérette à Ukegawa au cœur des montagnes des tranches de dauphin (photo 1), ce qui démontre la persistance de cette consommation locale. Cette chair comme celle de la baleine ont longtemps été la seule source de protéines pour les habitants de la côte, du fait de leur faible coût. L'image gastronomique de Taiji continue à s'appuyer sur la consommation de chair de baleine. En 2010 une feuille de promotion touristique donne la liste de 15 établissements où l'on peut consommer ce cétacé. Elle conclut que c'est sous forme de sashimi que cette viande se savoure le mieux et en second sous forme de chair fraîche hachée (tataki)... 
Photo 1 : Barquettes de chair de dauphin (iruka) dans une supérette à Ukegawa/Dolphin meat in a supermarket in Ukegawa

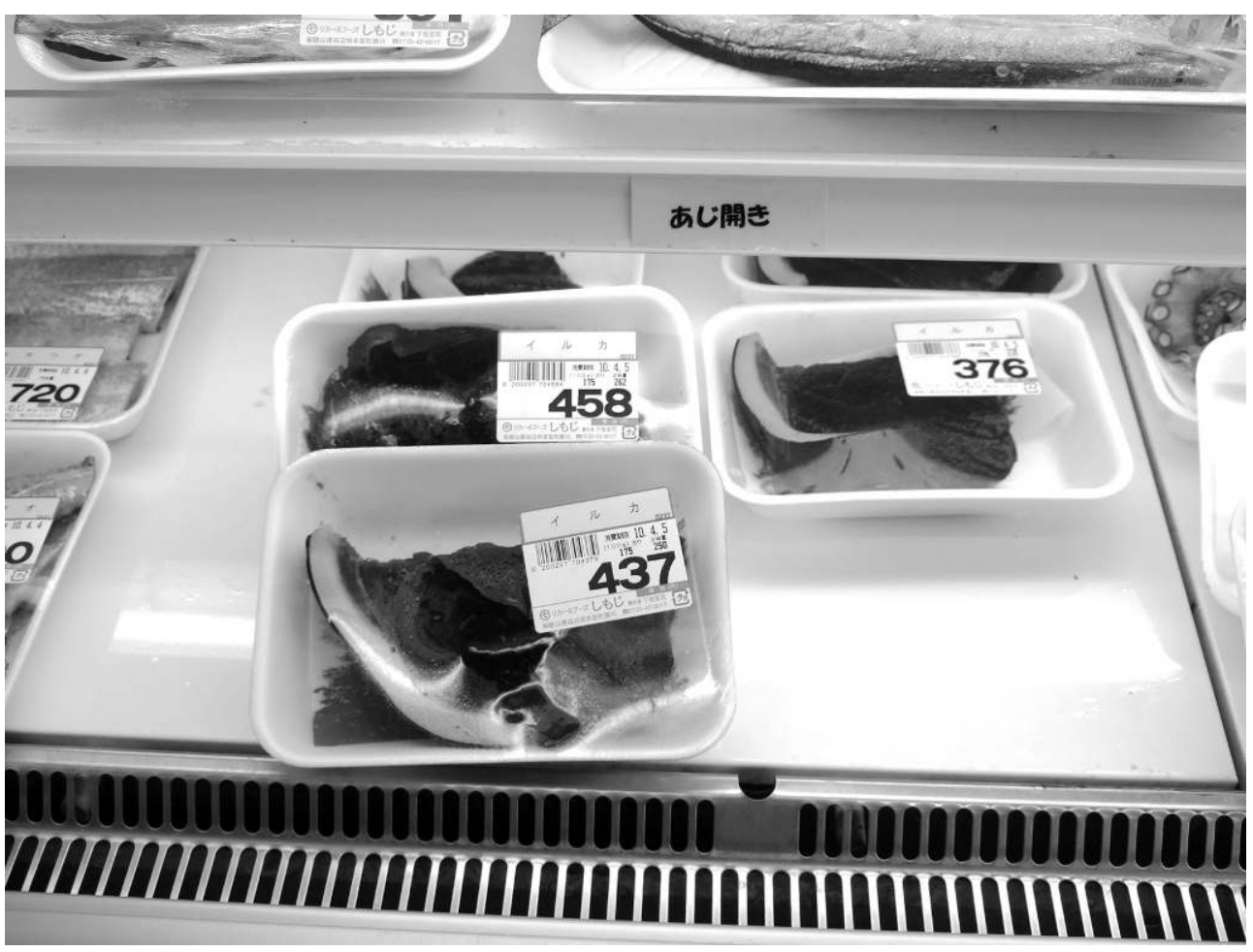

\section{Kii Tanabe : la culture des izakaya}

À l'intérieur de cet ensemble par quels produits se distingue la culture alimentaire de Kii Tanabe ? Commençons par renouer avec les douceurs évoquées ci-dessus. Kii Tanabe se trouve bien loin de figurer parmi les villes dont la réputation gourmande se fonde sur la présence de célèbres maisons de douceurs. Pourtant lors de la grande exposition nationale et quadriennale de douceurs qui s'est tenue à Himeji en 2008, c'est la maison Fumidō de Kii Tanabe qui a remporté le prix d'or, en d'autres termes le premier prix. Cette maison remonte à plus d'une centaine d'années et avait déjà obtenu le prix du ministre de l'Agriculture, de la forêt et de la pêche lors de cette même exposition en 2002. Elle a mis au point un sablé noir à la forme de charbon de type bichotan, une grande spécialité de Kii Tanabe, qui comporte du beurre, du sucre, des œufs, des grains de sésame noir et... de la poudre de charbon. Ce dernier, produit localement de façon manuelle, conserve la forme des troncs. Coupé en tronçons, il possède une excellente réputation pour faire griller les mets dans les restaurants japonais.

La maison Fumidō il y a une dizaine d'années a également recréé une douceur associée au pèlerinage de Kumano, les yama iwai mochi (littéralement les mochi qui fêtent les montagnes). Ils étaient consommés par les pèlerins à l'époque d'Edo, qui venus des lointaines contrées de l'est du Japon avaient réussi à accomplir leur périple sans encombre. Des riz rouge ou noir venant de l'antiquité japonaise mêlés à du riz blanc donnent leur couleur caractéristique à une enveloppe souple. À l'intérieur de ces douceurs, une pâte à base de sésame noir ou de citron japonais (yuzu Citrus Junos) procure un parfum particulier. Ces riz riches en minéraux, vitamines et fibres pourvoient ces douceurs de toutes sortes de qualités très appréciées de nos jours. 
Plusieurs produits du secteur primaire procurent à Kii Tanabe une réputation nationale. Il s'agit principalement pour l'agriculture des prunes (ume) et des mandarines (mikan) et pour la pêche, de la blanchaille (composée de minuscules sardines, shirasu). Tout d'abord envisageons les prunes de Kishū.

Le prunier introduit de Chine est autant apprécié pour sa floraison que pour ses fruits au Japon. Les pruniers porteurs de fruits comptent une centaine de variétés locales dans l'archipel, dont deux principales à Kii Tanabe (Gojirō et Nankō). À l'époque d'Edo leur culture a été encouragée sur les sols maigres exemptés de taxe dans le fief de Kishū, en particulier par le seigneur vassal local dont le château se trouvait à Kii Tanabe. Vers le milieu de cette époque avec le bois provenant de l'arrière-pays, le charbon bichotan sus mentionné et les mandarines, les prunes salées étaient expédiées vers la capitale d'Edo, l'actuelle Tokyo. Néanmoins leur qualité reste très inférieure à celle des prunes contemporaines. Leur production va cependant se développer considérablement du fait des vertus thérapeutiques qui leurs sont attribuées lors de grandes épidémies à l'époque de Meiji (1868-1912), des différentes guerres menées par le Japon. En effet les prunes salées se trouvent incorporées à l'ordinaire des soldats. De nouvelles variétés comme celles de Nankō et Gojirō mises au point au tournant du $\mathrm{XIX}^{\mathrm{e}}$ siècle et $\mathrm{au}$ début $\mathrm{du} \mathrm{xx}^{\mathrm{e}}$ siècle sont introduites, et la demande ne fait qu'augmenter. La consommation régulière de prunes salées avec le riz blanc s'intègre de façon idéale à la vogue d'une alimentation saine et naturelle à partir des années 1980. Consommées salées, ou sous forme de sauce, ou bien marinées dans le sake (umeshu) ou encore pressées en jus de fruits, la consommation de ces prunes ne cessent de se diversifier à la fin du $\mathrm{xx}^{\mathrm{e}}$ siècle.

Plantés en particulier sur les premières pentes des montagnes de Kumano, ces arbres offrent un paysage caractéristique de cette région avec les ventilateurs destinés à brasser l'air en cas de gel. Ils permettent une double récolte, avec en juin les fruits verts et un peu plus tard les mêmes murs. Vertes les prunes servent à préparer l'alcool aux prunes ou sont expédiées afin d'être transformées. Mûres, elles sont mises un mois dans le sel avant d'être exposées au soleil pendant 3 à 4 jours. Ce sont ces prunes salées qui font la réputation de Kii Tanabe (photo 2). Leur taille, leur souplesse, leur goût à la fois acide et doux en fait un excellent accompagnement du riz blanc qui n'est, il faut le rappeler, jamais salé. 
Photo 2 : Prunes salées en vente à Akinotsu/Sales of salted prunes in Akinostu

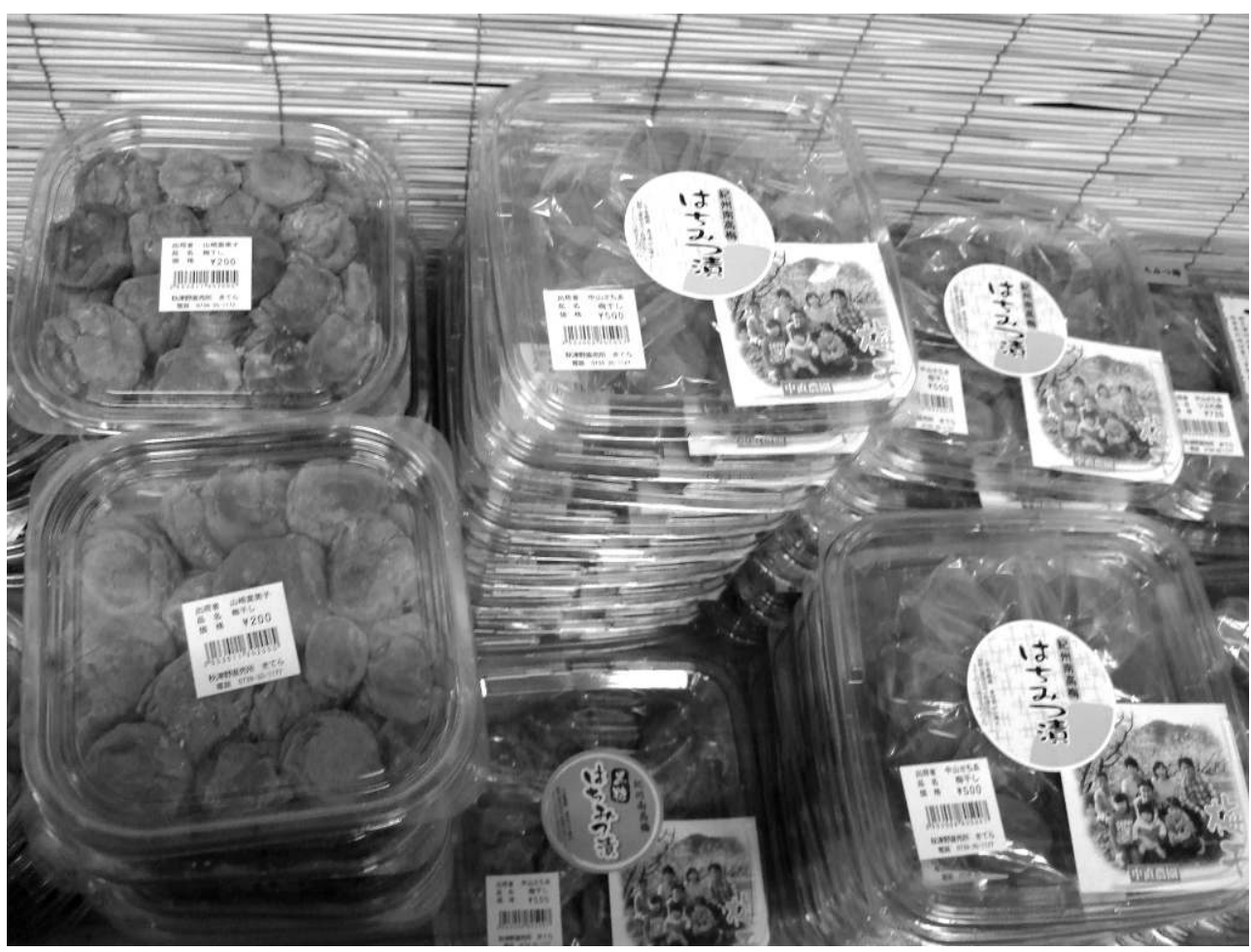

39 La confection des prunes salées a évolué en fonction de la modification de la demande de la part de consommateurs de plus en plus soucieux de la quantité de sel qu'ils absorbent quotidiennement. Cependant ce sel reste indispensable à leur conservation. Si sa quantité diminue il faut avoir recours à des conservateurs chimiques! Les coopératives agricoles ne cessent de promouvoir une consommation régulière de ces prunes, s'appuyant sur les bienfaits de leur consommation pour la santé (réduction du risque d'ulcère à l'estomac, amélioration de la pression artérielle, diminution du sucre dans le sang etc.). Également elles font la promotion de nouveaux modes de consommation, suggérant de nouvelles recettes et cherchant désormais à faire connaître ces fruits à l'étranger.

40 La production de prunes occupe le premier rang à Kii Tanabe, suivi par celle des mandarines. Toujours dans ce domaine cette ville se range à la première place dans le département de Wakayama. Ce dernier représente $27 \%$ des surfaces cultivées en pruniers de tout le Japon (sources municipales) et Kii Tanabe 7,6\% de cet ensemble. Le département produit $55 \%$ des prunes de type ume au Japon, avec 61600 tonnes parmi lesquelles 20600 proviennent de Kii Tanabe. Les exportations de l'industrie agroalimentaire représentent $60 \%$ du total des exportations industrielles à Kii Tanabe et celles de prunes correspondent à $80 \%$ de ce chiffre. Ces chiffres montrent à quel point ume est devenue emblématique de cette région.

Les mandariniers occupent le second rang de la production agricole de Kii Tanabe et disputent les pentes aux pruniers. Cette culture a été également encouragée à l'époque d'Edo. Leurs champs étagés représentent également un paysage caractéristique de ce département en particulier plus au nord. La récolte des mandarines (mikan) s'étale de septembre à décembre suivant les espèces qui sont fort nombreuses. Récemment les coopératives s'efforcent de diversifier l'offre de mikan sous formes de jus de fruits, de confitures etc. Ces fruits restent l'accompagnement inévitable de la consommation de 
thé vert en hiver, les jambes au chaud sous le brasero fixé dessous une table basse (kotatsu). Leur production s'élève à environ 19500 tonnes pour 913 hectares.

La baie de Kii Tanabe est à l'origine de plusieurs types de pêche et de préparations des produits de la pêche originaux, en particulier celui de la blanchaille de sardine (shirasu). Cette dernière doit faire moins de 2 à $3 \mathrm{~cm}$ de long (suivant les sources) pour conserver cette appellation. Deux barques permettent de tendre le filet destiné à prendre cette blanchaille (Wakayama Henshū Iinkai, 1989). Ramenée vers la surface de l'océan en douceur, elle conserve son intégralité et cuite tout de suite dans de grands chaudrons, garde sa fraîcheur, ce qui contribue à en faire un produit très recherché. Elle se nomme alors kama age shirasu (shirasu cuit à l'eau dans la marmite) et se distingue de chirimenjako ensuite séché au soleil. La blanchaille riche en calcium se consomme de manière variée, principalement sur du riz blanc. Le volume pêché ne cesse de diminuer depuis 1973 dans ces eaux baignées par le Kuro-shio si poissonneuses jadis. De 12700 tonnes en 1973 ce total est tombé à 5700 tonnes en 2007. Les différents types de sardines et de maquereaux représentent désormais la plus grande partie des prises, tandis que celle de shirasu s'élevait à 59 tonnes en 2007. Le port de pêche de Kii Tanabe connaît le même déclin que tous ceux de cette côte, si actifs et prospères pendant des siècles.

De quelle façon les restaurants dans Kii Tanabe valorisent-ils ces produits locaux? Force est de constater que la cuisine à base de produits de la mer reste dominante dans l'image donnée. Cependant de nouvelles tendances se dessinent qui cherchent à mettre en valeur les produits agricoles régionaux. Une toute récente parution élaborée avec le concours du bureau de tourisme départemental, centrée sur cette ville et intitulée Kumano kōdō (Les chemins de Kumano, 2010), se consacre à la culture alimentaire régionale. Le gruau de riz au thé, les prunes salées, les mikan, les mehari zushi, la blanchaille de sardines etc. se voient consacrés des pleines pages. Face à ces produits ou recettes qui ne datent pas d'aujourd'hui, plusieurs endroits qui offrent la possibilité de consommer des légumes produits localement trouvent aussi leur place, tendance nouvelle au Japon et à la mode. Ces lieux offrent une cuisine contemporaine ouverte sur des adaptations culinaires venues d'ailleurs.

Mais ce qui va retenir notre attention plus précisément ce sont les izakaya (lit. établissements ou l'on boit de l'alcool) et leur menus. Le quartier à la sortie de la gare présente un dédale de rues étroites ornées d'enseignes, vides dans la journée (photo 3) et qui le soir prend un aspect beaucoup plus animé. Ces établissements qui ne laissent rien deviner à l'extérieur se laissent découvrir le soir, une fois la porte franchie. La cuisine à base de produits de la mer y règne. Ces établissements n'affichent aucun menu à l'extérieur, ni parfois à l'intérieur et ils s'adressent à des habitués.

Le bureau de tourisme de Kii Tanabe nous a affirmé qu'un seul établissement dépend d'une chaîne, tous les autres appartenant à des personnes de la région tant cette culture locale reste forte. L'excellente qualité des produits, leur coût modique comparé à ceux des très grandes villes a donné l'idée à ce bureau de mettre plus en valeur cet aspect inébranlable de la culture alimentaire de Kii Tanabe. L'occasion fut donnée par la réunion internationale de Aïkido (son fondateur Ueshiba Morihei est un enfant du pays) qui se tenait en 2008 dans la ville. Le bureau de tourisme a établi une sélection d'établissements qui acceptaient pour la première fois d'utiliser l'anglais pour présenter quelques uns de leurs plats. En outre il a eu l'idée de créer le Ogara don (le bol de riz blanc garni «bien de chez nous» en dialecte de Kishū), qui n'utilisent que des 
produits locaux et diffèrent dans chaque établissement (photo 4). Dix-sept établissements ont accepté de se lancer dans cette aventure unique au Japon. Cette initiative permet aux étrangers dont le nombre grossit lentement, venus marcher sur les chemins de Kumano, d'aborder la cuisine locale, qui serait restée impénétrable avec le barrage de la langue, sans compter celui des habitudes.

Sans développer ici la polémique surgie au Japon à propos de la conception du guide Michelin qui démontre à quel point la fréquentation des restaurants diffèrent suivant les cultures, il faut souligner cette différence fondamentale. La plupart des établissements au Japon possèdent leurs propres habitués et n'éprouvent pas le besoin d'être affichés dans un guide, cela d'autant plus que leur taille ne permettrait pas une clientèle supplémentaire. La variété et le nombre établissent la renommée gastronomique d'un lieu... même si le regard occidental est en train d'établir une hiérarchie au sein de cette multitude.

Photo 3 : Le quartier des izaka-ya à Kii Tanabe/The izakaya-place in Kii Tanabe

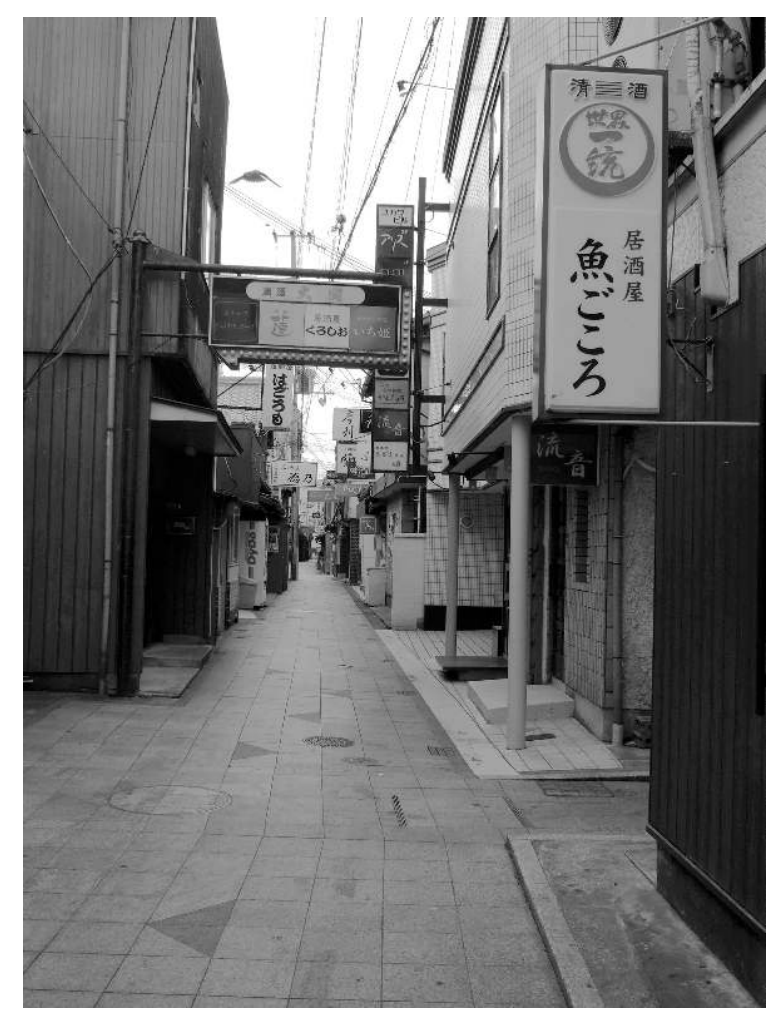


Photo 4 : Okara-don de chez Aji yōshi (litt. Au goût satisfaisant)/Okara-don from Aji yōshi ("Sastifiying taste")

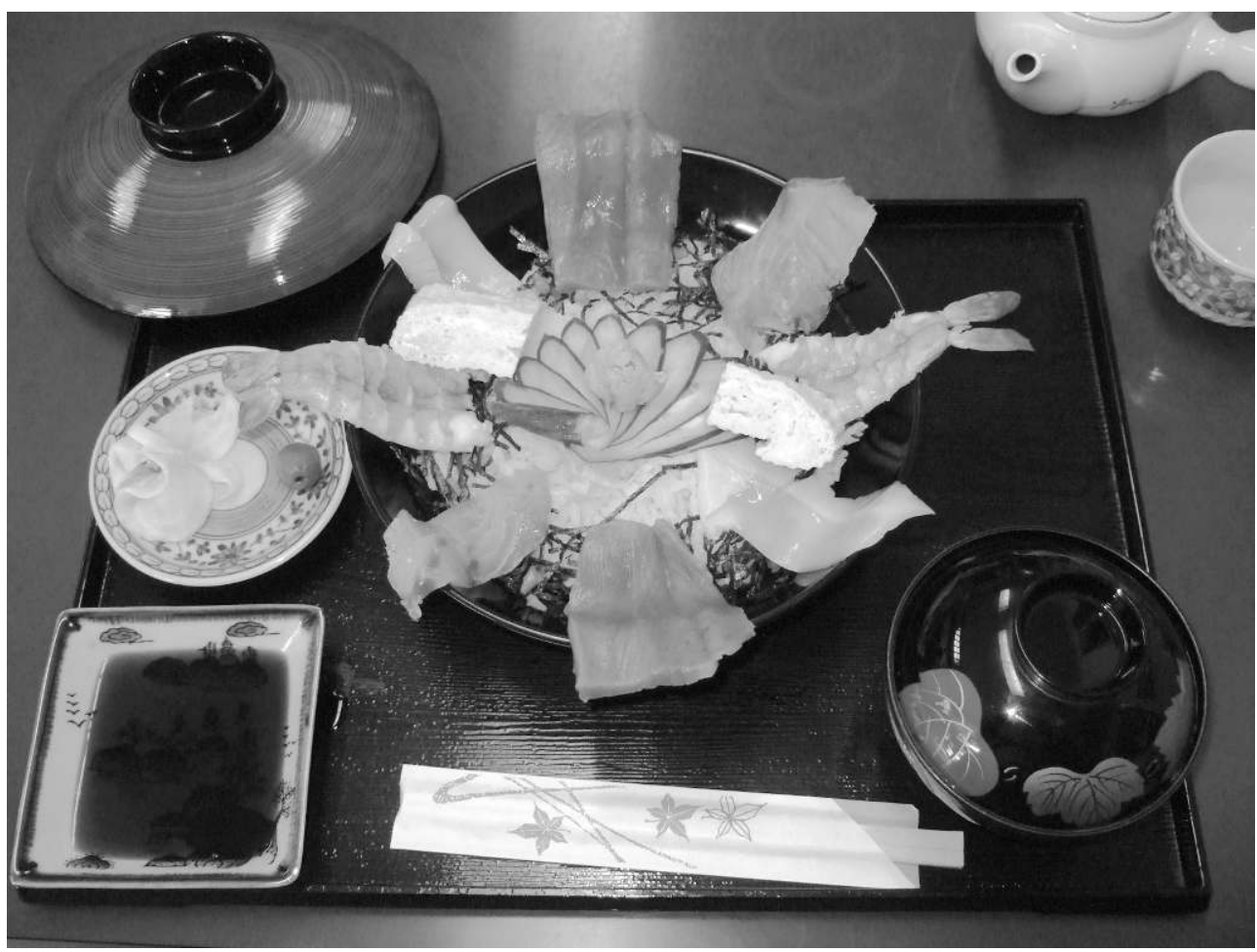

\section{Conclusion}

47 La région de Kii Tanabe ne possède pas de grand restaurant tel que la France a su en faire la promotion dans le monde, ni le reste du Japon d'ailleurs. Grâce à sa culture culinaire c'est la ville tout entière, voire la région qui constitue un lieu gourmand de renom où le voyageur vient goûter ici où là quelques-unes des spécialités locales, comme partout dans l'archipel. La richesse gastronomique régionale ne peut se faire interprétée par un seul établissement. Eternel voyageur le visiteur japonais découvre l'extraordinaire variété de la cuisine locale de son pays, qui ne cesse de s'enrichir d'apports extérieurs comme il le fait depuis des siècles.

\section{BIBLIOGRAPHIE}

ASAHI S., 7 novembre 2009. Tomaru nara ryokan? Hoteru ? (En matière d'hébergement ryokan ou hotel ?), n.p.

AshKENAZY M., JACOB J., 2000. The Essence of Japanese Cuisine, Richmond, Curzon, 252 p.

Les chemins de Kumano (Kumano kōdō), Tokyo, Diamond-Big, coll. « Chikyu no arukikata », 2010, 127 p. 
GUICHARD-ANGUIS S., (à paraître). « Rosée du matin » et « Matin d'hiver » : les douceurs (wagashi) dans le patrimoine culturel japonais, dans CSERGO J., et LEMASSON J.-P, Patrimoines alimentaires et destinations touristiques : passion ou raison ?, Lyon, XXII ${ }^{\mathrm{e}}$ Entretiens du Centre Jacques Cartier, 2009.

GUICHARD-ANGUIS S., 2008a. Introduction: The Culture of Travel and Japanese Tourism, dans GUICHARD-ANGUIS S., Moon O. (dir.), Japanese Tourism and the Culture of Travel, Londres/New York, Routledge, coll. «Japan Anthropology Workshop Series », p. 1-16.

GUICHARD-ANGUIS S., 2008b. Japanese inns (ryokan) as producers of Japanese Identity, dans GuICHARD-ANGUIS S., Moon O. (dir.), Japanese Tourism and the Culture of Travel, Routledge, Londres/ New York, coll. «Japan Anthropology Workshop Series », p. 76-101.

GUICHARD-ANGUIS S., 2003a. L'image de l'hébergement de luxe et l'hôtel, dans une revue féminine japonaise, dans FIÉVÉ N., GED F., GELÉZEAU V., GUICHARD-ANGUIS S., SANJUAN T., Les grands hôtels en Asie, Modernité, dynamiques urbaines et sociabilité, Paris, Publications de la Sorbonne, p. 169-189. GuICHARD-ANGUIS S., 2003b. Feuilles d'érables, terres cuites et poissons grillés. Une alternative de la sociabilité offerte par les ryokan au Japon, dans FIÉVÉ N., GED F., GELÉZEAU V., GUICHARD-ANGUIS S., SANJUAn T., Les grands hôtels en Asie, Modernité, dynamiques urbaines et sociabilité, Paris, Publications de la Sorbonne, p. 217-251.

JIPPENSHĀ I., 1992. À pied sur le Tōkaidō, (1806) Tōkaidōchu hizakurige, trans : CAMPIGNON J., Arles, Philippe Picquier, 393 p.

KANZAKI N., 2002. Tabi no hattatsu Shokuji no jujitsu (Essor du voyage Elaboration des repas), dans KANZAKI N. (dir.), Tabi to shoku (Voyage et alimentation), Tokyo, Domesu shuppan, p. 241-263.

KANZAKI N., 1997. Omiyage (Cadeaux), Tokyo, Seikyūsha, 222 p.

KARIYA T., HANASAKI A. 2009. Oishinbo 103 Nihon Zenken aji mawari Wakayama hen (Gourmet de gourmandises 103 Tour de tous les départements Wakayama), Tokyo, Shogakan, coll. « Biggu komikkusu », 250 p.

KoYAMA Y., 2004 (2006). Yoshino. Koya. Kumano o yuku Reijō to sankei no michi (Se rendre à Yoshino. Koya. Kumano Lieux sacrés et chemins de pèlerinage), Tokyo, Asahi shimbumsha, coll. « Asahi sensho », $182 \mathrm{p}$.

KoYAMA Y., 2000. Kumano kodō (Les anciens itinéraires de Kumano), Tokyo, Iwanami, coll. «Iwanami shinsho », 208 p.

Kozu A., 2009. Chanoyu no rekishi (Histoire de Chanoyu), Tokyo, Kadokawa, 286 p.

MoERMAN D. M., 2006. Localizing Paradise: Kumano Pilgrimage and the Religious Landscapes of Premodern Japan, Cambridge, Massachusetts, Harvard University Asia Center, 297 p.

TORAYA BUNKo, 2006. "Wagashi de tanoshimu dōchū nikki" Ten (Exposition sur des « Journeaux de voyage dont on peut savourer les douceurs »), Toraya, Tokyo, $40 \mathrm{p}$.

TANIGUCHI J., KuSUMI M., 2005. Le gourmet solitaire (Kudoku no gurume), Paris, Casterman, 198 p. VAPORIS N. C., 2008. Tour of Duty, Honolulu, University of Hawai 'i Press, 318 p.

WAKAYAMA HENSHŪ IINKAI, 1989. Nihon no shoku seikatsu zenshū, vol. 30 Wakayama no shokuji (Encyclopédie sur la culture alimentaire du Japon, vol. 30 Les repas de Wakayama), Tokyo, Nōzan gyoson bunka kyokai, n.p. 


\section{NOTES}

1. [http://www.tsuentea.com/jpindex.htm].

2. [http://www.akafuku.co.jp/index.html].

3. [http//b-tsuyama.com].

4. Voir à ce propos Hōsei daigaku gendai fukushi gakubu Nakamura kenkyushistu, Kansai gakuin daigaku shakai gakubu Furukawa kenkyūshitsu, Kyoto daigaku bungakubu shakaigaku kyōshitu. 2001. -Chiiki ni manabu (Ce que nous apprend la région. Vol. 6, n. p.).

5. Les mochi sont des gâteaux de riz fait à partir de riz gluant cuit à la vapeur et écrasé au pilon.

\section{RÉSUMÉS}

À Kii Tanabe (Japon) ou même dans toute la péninsule de Kii, on ne trouve pas de grand restaurant tel qu'on les conçoit en France. Pourtant la richesse gustative de cette petite ville, sise loin des grands foyers urbains est incontestable. Elle reste exemplaire de ce que l'on peut trouver un peu partout au Japon. Elle reflète l'histoire locale et tire parti de toutes les ressources régionales, pour satisfaire une clientèle essentiellement cantonnée à cette ville même et ses alentours. Depuis quelques années cette cuisine tente de s'adresser à une clientèle nationale, voire internationale avec la nomination au patrimoine mondial des chemins de pèlerinage de Kumano, pour lesquels Kii Tanabe joue le rôle de porte d'entrée.

In Kii Tanabe or even in the Kii peninsula, no famous restaurant as the ones which can be found in French countryside can be named. In spite of this, the richness of the culinary culture of this small city, located far away from the large urban regions cannot be denied. It gives a very good example of what can be found all over Japan. This culinary culture reflects regional history and takes advantage of all the local ingredients, in order to satisfy local consumers. With the nomination of the pilgrimage roads of Kumano in the Unesco list of world culture heritage, Kii Tanabe became the door to this tourist attraction. Since a few years the local cuisine is aiming at satisfying consumers coming from all over Japan and even abroad.

\section{INDEX}

Index géographique : Japon

Mots-clés : gastronomie, spécialités, culture culinaire, Kii Tanabe

Keywords : gastronomy, specialities, culinary culture, Kii Tanabe 


\section{AUTEUR}

\section{SYLVIE GUICHARD-ANGUIS}

Laboratoire Espaces, Nature et Culture (ENeC) UMR 8185 CNRS (Université Paris 4 - Sorbonne), 190-198 avenue de France - 75013 Paris, France Sguichard_anguis@hotmail.com 